\title{
Promoção da saúde, prevenção de doenças e utilização de serviços: avaliação das ações de uma operadora de plano de saúde brasileira
}

\author{
Health promotion, disease prevention, and health service \\ use: evaluation of the actions of a Brazilian private health plan operator
}

\author{
Danielle Conte Alves ${ }^{1}$, Maria Alicia Dominguez Ugá², \\ Margareth Crisóstomo Portela ${ }^{2}$
}

\begin{abstract}
Resumo
Introdução: Trata-se de estudo de caso que teve como objetivo explorar a associação entre ações de promoção da saúde e prevenção de doenças cardiovasculares (DC) desenvolvidas por uma operadora de plano de saúde brasileira que adotou a Estratégia Saúde da Família, a utilização de serviços e os respectivos custos. Métodos: Foram selecionados os beneficiários com idade maior ou igual a 30 anos, cadastrados nos programas até 31 de dezembro de 2004 em duas capitais. Foi realizada a descrição do perfil demográfico e dos fatores de risco para DC, bem como a avaliação do perfil de utilização de serviços e dos custos, entre 2004 e 2007. Resultados: Verificou-se a redução do número médio de consultas por beneficiário e do custo médio com essas consultas no grupo cadastrado. O número de exames per capita aumentou, elevando esses custos. Em Curitiba, no Paraná, ocorreu aumento do número médio de internações por beneficiário e do custo médio, enquanto, em Goiânia, Goiás, ocorreu redução desses números. Conclusão: Considerando que cerca de $25 \%$ da população brasileira possui plano de saúde, torna-se relevante incentivar a realização desses programas, em consonância com as políticas empreendidas pelo Ministério da Saúde (MS), além de avaliar a utilização dos serviços e os resultados na saúde dos indivíduos.
\end{abstract}

Palavras-chave: promoção da saúde; prevenção de doenças; saúde suplementar; planos de saúde; serviços de saúde.

\begin{abstract}
Introduction: This article describes a case study which aimed to explore the association between health promotion actions and the prevention of cardiovascular diseases (CD). These actions were developed by a Brazilian health maintenance organization that has adopted the Family Health Strategy and the use of health services and their related costs. Methods: The method involved the selection of beneficiaries from two capitals, aged 30 years or older, who subscribed until Dec 31, 2004. A description of the demographic profile and risk factors for CD was performed, as well as an evaluation of the profile of use of services and their corresponding costs between 2004 and 2007. Results: There was a reduction in the average number of appointments per beneficiary and in the average cost of these appointments. There was an increase in the number of exams per capita, increasing assistance costs. In Curitiba, there was an increase in the average number of hospitalizations per beneficiary and in their average cost, whereas in Goiania a reduction of such numbers was observed. Conclusion: Considering that approximately $25 \%$ of the Brazilian population is currently covered by health maintenance organizations, it is important to encourage the adoption of these programs, in agreement with the policies undertaken by the Ministry of Health, in addition to evaluating the use of health services and the outcomes in the health of individuals.
\end{abstract}

Keywords: health promotion; disease prevention; supplemental health; prepaid health plans; health services.

\footnotetext{
${ }^{1}$ Agência Nacional de Saúde Suplementar (ANS) - Rio de Janeiro (RJ), Brasil.

${ }^{2}$ Escola Nacional de Saúde Pública Sergio Arouca (ENSP), Fundação Oswaldo Cruz (FIOCRUZ) - Rio de Janeiro (RJ), Brasil.

Trabalho realizado na Escola Nacional de Saúde Pública Sergio Arouca (ENSP), Fundação Oswaldo Cruz (FIOCRUZ) - Rio de Janeiro (RJ), Brasil.

Endereço para correspondência: Danielle Conte Alves - Av. Augusto Severo, 84, Edifício Barão de Mauá - Glória - CEP: 20021-040 - Rio de Janeiro (RJ), Brasil -

Email: danielle.conte@gmail.com
}

Fonte de financiamento: próprio.

Conflito de interesses: nada a declarar. 


\section{INTRODUÇÃO}

O debate em torno da promoção da saúde influencia as políticas públicas de distintos países, tendo em vista o questionamento do paradigma biomédico e a mudança do perfil epidemiológico e demográfico da população nas últimas décadas. Isso ensejou o surgimento de propostas em torno da reorientação de um modelo de atenção voltado para práticas preventivas e para a integralidade do cuidado em saúde.

No que tange à regulação do setor privado suplementar de assistência à saúde, o tema da promoção da saúde e da prevenção de doenças foi colocado em pauta pela Agência Nacional de Saúde Suplementar (ANS) em 2005, a partir da publicação da Resolução Normativa $n^{\circ} 94$, que estabeleceu incentivos para as operadoras de planos de assistência à saúde que desenvolvessem programas de promoção da saúde e de prevenção de doenças ${ }^{1,2}$.

Desde então, a ANS vem incentivando a adoção desses programas pelas operadoras por meio da instituição de novos normativos $^{3-8}$ e de publicações como: Manual técnico de promoção da saúde e prevenção de riscos e doenças na saúde suplementar ${ }^{9}$, Modelagem de programas ${ }^{10}$, Plano de cuidado para idosos na saúde suplementar ${ }^{11}$, Guia e cartão sobre plano de melhoria da saúde ${ }^{12,13}$, bem como publicações que abordam programas desenvolvidos nas áreas de odontologia e oncologia ${ }^{14,15}$, e publicação que descreve os perfis dos programas desenvolvidos na saúde suplementar ${ }^{16}$.

Ademais, dentre os temas prioritários da Agenda Regulatória da ANS para o biênio 2013-2014, foram estabelecidos os projetos: "Estudar e incentivar a adoção, pelas operadoras de planos de saúde, de modelo assistencial centrado no Plano de Cuidado" e "Estudar alternativas para permitir/estimular o incentivo financeiro nas ações de promoção e prevenção"17.

Vários fatores contribuem para o aumento constante dos custos em saúde, como o envelhecimento populacional, o perfil de morbimortalidade da população, com o aumento das doenças crônicas não transmissíveis (DCNT) ${ }^{18}$, e a incorporação de tecnologias que, na produção de serviços de saúde, não substituem, mas frequentemente se somam às previamente vigentes ${ }^{19}$. Na saúde suplementar, a regulamentação do setor, a partir da Lei no 9.656 , de 1998, trouxe importantes avanços regulatórios, alguns dos quais contribuíram para a elevação dos custos, como a ampliação de coberturas e a inclusão de tratamento para $\mathrm{DCNT}^{20}$.

Embora prestem serviços na área da saúde, as empresas de planos de saúde são regidas pela lógica de mercado. No entanto, é necessário conciliar esse objetivo com as políticas empreendidas pelo Ministério da Saúde (MS), respeitando as particularidades do setor, a fim de aumentar a qualidade da atenção à saúde.

As operadoras de planos privados de saúde médico-hospitalares são classificadas pela ANS nos seguintes grupos: administradora de planos, administradora de benefícios, autogestão, cooperativa médica, filantropia, medicina de grupo e seguradora especializada em saúde ${ }^{21}$. A autogestão é a modalidade de assistência privada à saúde na qual a própria empresa ou organização administra o programa de assistência à saúde dos seus empregados e dependentes $^{22,23}$.

Do total de 50,3 milhões de beneficiários em planos de assistência médica com ou sem odontologia existentes no país em setembro de 2015, as operadoras de autogestão abrangiam 5,5 milhões desses beneficiários, conforme dados do Sistema de Informação de Beneficiários da $\mathrm{ANS}^{21}$. Uma das características dessa modalidade é não visar ao lucro. Os serviços médicos podem ser próprios, de rede credenciada ou do sistema de livre escolha, por meio de reembolso ${ }^{22,24}$.

Segundo a União Nacional das Instituições de Autogestão em Saúde (UNIDAS), os investimentos em medicina preventiva aumentaram entre as empresas de autogestão, as quais, por terem uma clientela relativamente estável, têm a capacidade de planejar e implementar ações visando à redução de gastos com assistência médico-hospitalar, promovendo, ao mesmo tempo, melhorias na qualidade de vida dos seus beneficiários ${ }^{24}$.

O objetivo do presente estudo foi explorar a associação entre a realização de ações de promoção da saúde e prevenção de doenças cardiovasculares (DC) e o uso de serviços de saúde (consultas ambulatoriais; serviços de apoio à diagnose e terapia; internações), bem como os custos correspondentes na atenção à saúde dos beneficiários de uma operadora de plano de saúde, pertencente à modalidade de autogestão, que adotou a Estratégia Saúde da Família (ESF).

\section{MÉTODOS}

Coombes e Thorogood ${ }^{25}$ ressaltam que não há somente um método para a avaliação na área de promoção da saúde e prevenção de doenças, uma vez que cada tipo de atividade em promoção da saúde demanda uma diferente forma de avaliar, necessitando, assim, de métodos rigorosos de avaliação capazes de sustentar as conclusões obtidas.

Este é um estudo de caso, de caráter descritivo, no qual foram selecionados os beneficiários associados à operadora de plano de saúde estudada provenientes de duas capitais, Curitiba, no Paraná, e Goiânia, em Goiás, com idade maior ou igual a 30 anos, cadastrados até 31 de dezembro de 2004 nos programas com ações voltadas para promoção da saúde e prevenção de DC, quais sejam: programa para indivíduos com 60 anos ou mais, programa de assistência domiciliar e programa para portadores de DCNT.

Optou-se pela realização do estudo de caso em uma empresa de autogestão, tendo em vista ser maior a chance de permanência do beneficiário no plano por um longo período de tempo, o que permite acompanhar a evolução de um programa de promoção e de prevenção. 
Segundo Hartz, no que se refere à validação externa, os estudos de caso têm o interesse de generalizar um quadro teórico ou modelo relacionado à compreensão de um determinado problema em diferentes situações, sem a pretensão de alcançar uma generalização estatística ${ }^{26}$.

Primeiramente, realizou-se a descrição do perfil demográfico dos indivíduos selecionados para a pesquisa, além do perfil de saúde e dos fatores de risco para DC. Essas informações foram obtidas por meio dos registros no prontuário eletrônico da operadora, o qual permite conhecer o perfil de fatores de risco nessa população, tais como: consumo excessivo de álcool, dislipidemia, sedentarismo, tabagismo, obesidade, hipertensão arterial sistêmica (HAS) e diabetes mellitus (DM) ${ }^{27,28}$.

Em seguida, foi realizada a avaliação do perfil de utilização de serviços de saúde (consultas ambulatoriais; serviços de apoio à diagnose e terapia; internações) por esses beneficiários cadastrados, com os correspondentes custos na prestação da assistência à saúde, no período de 2004 a 2007.

A base de dados, cedida pela própria empresa, contou com as seguintes variáveis: sexo, data de nascimento, consultas, internações, exames e procedimentos clínicos ambulatoriais realizados, além dos valores pagos por cada procedimento.

O Índice Geral de Preços - Disponibilidade Interna (IGP-DI) ${ }^{29}$ foi utilizado para fins de comparação dos custos com consultas ambulatoriais, internações, exames e procedimentos clínicos ambulatoriais entre os quatro anos de análise. Para tanto, foram considerados os valores médios anuais para 2004 e 2005, e os valores de dezembro para os anos de 2006 e 2007 - este último ano foi utilizado como referência para a deflação dos valores dos anos anteriores.

\section{Perfil de utilização de consultas}

No que concerne à análise da base de dados de consultas, foram selecionadas as consultas com clínico geral, cardiologista, endocrinologista e nutricionista. A escolha justifica-se por serem esses profissionais os mais envolvidos em programas de prevenção de DC. Não foram encontrados registros de outros profissionais de saúde na base de dados em questão.

\section{Perfil de utilização de exames e procedimentos selecionados}

A fim de verificar o perfil de utilização de exames e procedimentos, foram selecionados os exames listados na Programação Pactuada Integrada (PPI) do MS, no que se refere aos programas voltados para a saúde do adulto e idoso, com foco em HAS e $\mathrm{DM}^{30}$, assim como os procedimentos clínicos ambulatoriais, subitem "monitorizações", e os procedimentos diagnósticos e terapêuticos constantes na Classificação Brasileira Hierarquizada de Procedimentos Médicos (CBHPM) da Associação Médica Brasileira ${ }^{31}$, conforme os itens listados a seguir:
- Exames PPI - ácido úrico, clearance de creatinina, colesterol total, HDL-c, LDL-c, creatinina, eletrocardiograma (ECG), glicose, hemoglobina glicosilada, hemograma, lipidograma completo, microalbuminúria, potássio, EAS e triglicerídeos;

- Procedimentos clínicos ambulatoriais CBHPM - holter-24 horas, MAPA-24 horas e tilt teste; e

- Procedimentos diagnósticos e terapêuticos CBHPM - ECG (também indicado pela PPI), ergoespirometria ou teste cardiopulmonar de exercício completo e teste ergométrico.

\section{Perfil de utilização de internações por causas selecionadas}

Para fins de análise das internações entre os beneficiários cadastrados, foram selecionadas as causas de internação por DC e por doenças relacionadas ao alto risco de eventos coronários, além da realização de procedimento cirúrgico. As causas de internação pela clínica médica ou cirúrgica selecionadas foram: acidente vascular cerebral (AVC); crise hipertensiva; DM; hipertensão maligna; infarto agudo do miocárdio (IAM); insuficiência cardíaca; insuficiência coronariana aguda; insuficiência renal crônica; revascularização do miocárdio; tratamento da obesidade; cirurgia de obesidade mórbida.

Impende ressaltar que, por motivos de registro, não foi possível utilizar a Classificação Internacional de Doenças (CID) para a seleção das causas de internação, o que levou à opção de selecionar as causas descritas na base de dados fornecida pela operadora de plano de saúde. Sabe-se que, dessa forma, alguns dados podem estar subestimados, tendo em vista que muitas internações para realização de procedimentos cirúrgicos podem ter sido motivadas por DC ou pelas demais doenças selecionadas, não identificadas na base pelo não preenchimento da CID.

Após a seleção das causas de internação para análise, observou-se que a internação de um mesmo beneficiário poderia aparecer em mais de um registro na base de dados, com valores de pagamento diferentes, devido às diferentes guias emitidas durante a internação, fato que dificultou a identificação do número de internações de cada beneficiário e do custo total de cada internação. Para tanto, os registros dos beneficiários e os correspondentes valores foram considerados como provenientes de uma única internação quando as datas de pagamento possuíam o intervalo de, no máximo, sete dias.

\section{Análise estatística}

Foi utilizado o teste não paramétrico de Wilcoxon para a comparação das médias de variáveis numéricas, como as que discriminam a média do número de consultas, exames e internações, bem como os custos assistenciais.

Utilizou-se o pacote estatístico SAS ${ }^{\circledast}$. 


\section{Comitê de Ética em Pesquisa}

A realização da pesquisa foi aprovada pelo Comitê de Ética em Pesquisa da Escola Nacional de Saúde Pública Sergio Arouca (CEP/ENSP), por meio do Protocolo de Pesquisa no 108/08.

\section{RESULTADOS}

A estrutura do programa de promoção da saúde e prevenção de doenças desenvolvido pela operadora estudada está baseada na adoção de serviços próprios e na contratação de equipes multidisciplinares, que têm sob sua responsabilidade um determinado número de beneficiários para coordenação dos cuidados em saúde. Essa prática viabiliza o conhecimento das características sociais, demográficas e epidemiológicas da população cadastrada, com a identificação dos problemas de saúde prevalentes e situações de risco.

\section{Análise descritiva da amostra de beneficiários selecionados}

A partir dos critérios estabelecidos, foram selecionados 9.966 beneficiários com idade igual ou maior que 30 anos da operadora de plano de saúde estudada, dos quais 3.900 (39,1\%) eram beneficiários cadastrados. Desse total, $68,2 \%(n=2.658)$ eram de Curitiba, e 31,8\% ( $n=1.242)$, de Goiânia.

Quanto à distribuição dos beneficiários cadastrados de acordo com o sexo, os percentuais de indivíduos do sexo masculino foram de 44,3\% ( $n=1.178)$ em Curitiba e 46,9\% ( $n=583)$ em Goiânia, enquanto, entre as mulheres, esses percentuais foram de $55,7 \%(n=1480)$ e $53,1 \%(n=659)$, respectivamente.

Dentre os cadastrados de Curitiba, 75,1\% ( $\mathrm{n}=1995)$ possuíam idade entre 30 e 59 anos, enquanto $24,9 \%(n=663)$ possuíam 60 anos ou mais. Em Goiânia, tais percentuais foram de 70,8\% $(\mathrm{n}=879)$ e $29,2 \%(\mathrm{n}=363)$, respectivamente.

\section{Análise descritiva do perfil de saúde dos beneficiários}

Ao analisar o perfil dos fatores de risco para DC, verificou-se que, em Curitiba, os mais frequentes entre os beneficiários com idade entre 30 e 59 foram dislipidemia (12,7\%), HAS $(9,5 \%)$ e obesidade (8,0\%), enquanto os mais frequentes entre os indivíduos com 60 anos ou mais foram HAS (29,0\%), dislipidemia (17,3\%) e DM (9,2\%) (Gráfico 1).

Em Goiânia, os fatores de risco mais frequentes entre os indivíduos com idade entre 30 e 59 anos foram sedentarismo $(38,2 \%)$, dislipidemia ( $14,7 \%)$ e obesidade (13,9\%), enquanto que, entre os beneficiários com 60 anos ou mais, os mais frequentes foram HAS $(40,2 \%)$, sedentarismo $(34,7 \%)$ e dislipidemia (25,3\%) (Gráfico 2).

\section{Análise da utilização de consultas}

Apresenta-se, a seguir, a análise da utilização de consultas com clínico geral, cardiologista, endocrinologista e nutricionista em cada ano estudado, pelos cadastrados nos programas,

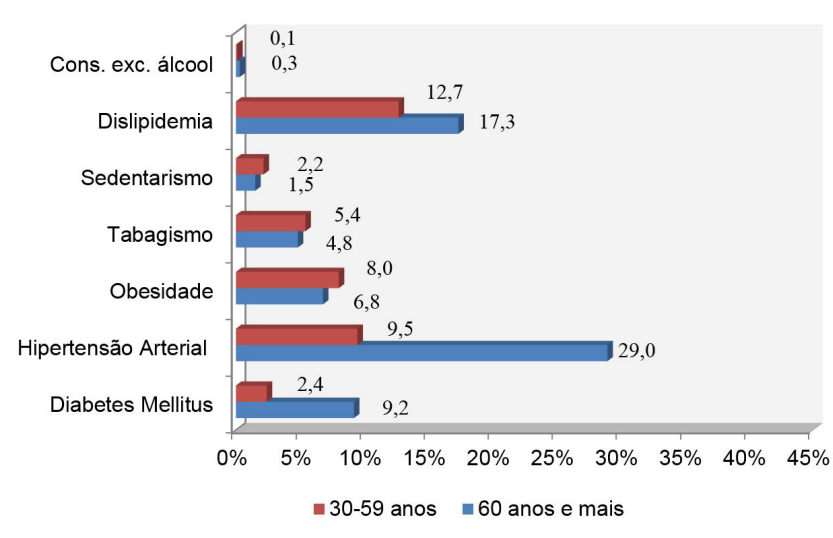

Gráfico 1. Distribuição percentual dos fatores de risco para doenças cardiovasculares ${ }^{27,28}$ entre os beneficiários cadastrados em programas de promoção da saúde e prevenção de doenças cardiovasculares, em Curitiba, de acordo com a idade

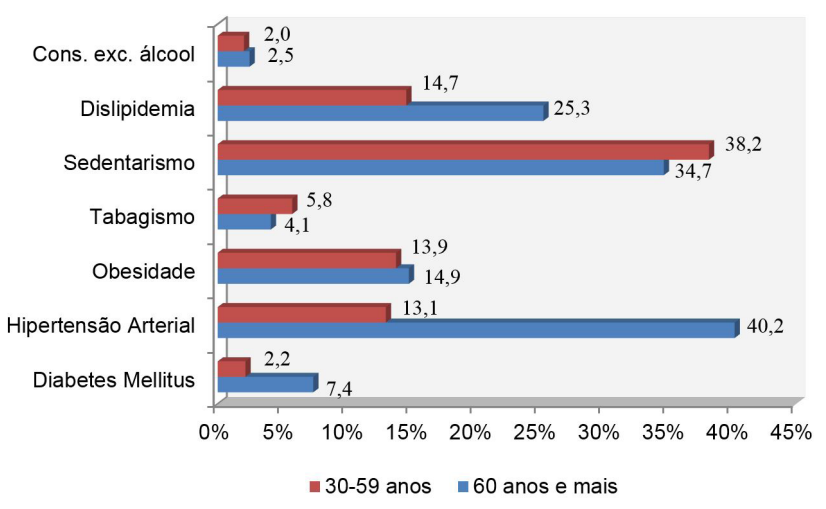

Gráfico 2. Distribuição percentual dos fatores de risco para doenças cardiovasculare $\mathrm{s}^{27,28}$ entre os beneficiários cadastrados em programas de promoção da saúde e prevenção de doenças cardiovasculares, em Goiânia, de acordo com a idade

em relação ao número total de beneficiários cadastrados nos programas para prevenção de DC, com idade maior ou igual a 30 anos, no respectivo ano.

A média do número de consultas por beneficiário cadastrado que realizou alguma consulta, em Curitiba, diminuiu entre 2004 e 2007. Em 2006, mesmo com o aumento do número total de consultas, a média per capita foi menor em comparação ao ano anterior. No que diz respeito à análise do custo médio por beneficiário, ocorreu elevação dos gastos com consultas entre 2004 e 2006, com redução em 2007 (Tabela 1).

Em Goiânia, a média do número de consultas e o custo médio por beneficiário aumentaram em 2005 em relação a 2004, apresentando redução a partir de 2006. Cabe destacar a grande redução do número de consultas em 2007, o que também se refletiu na média de consultas per capita e no custo médio (Tabela 1).

Em ambas as unidades da operadora estudada, o número total de consultas diminuiu entre os anos de 2004 a 2007, 
bem como o número médio de consultas por beneficiário. Em Goiânia, houve um aumento da média per capita em 2005 e, em Curitiba, ocorreu aumento do número total de consultas em 2006, porém, nos anos seguintes, esses valores tornaram a diminuir. No que diz respeito à análise do custo médio com consultas por beneficiário, ocorreu redução dos gastos entre 2004 e 2007 (Tabela 1).

O teste estatístico evidenciou que a redução da média de consultas per capita no período analisado foi bastante significativa, assim como os resultados obtidos quanto ao custo médio com consultas por beneficiário, tanto em Curitiba quanto em Goiânia (p-valor $<0,001$ ) (Tabela 1).

\section{Análise da utilização de exames e procedimentos selecio- nados}

No período de 2004 a 2006, observou-se o aumento do número total de exames e da média de exames utilizados por beneficiário cadastrado que utilizou algum exame em Curitiba, com o consequente aumento do custo. Esses valores apresentaram redução em 2007, ainda assim a média do número de exames por beneficiário e o custo assistencial com os exames e procedimentos selecionados continuaram mais elevados em comparação ao ano de 2004 (Tabela 2).

A média do número de exames e procedimentos selecionados realizados por beneficiário cadastrado em Goiânia aumentou entre 2004 e 2006 (19,06 e 23,43, respectivamente), o que se refletiu no aumento do custo médio por beneficiário no período em questão (Tabela 2).

$\mathrm{Na}$ análise dos exames e procedimentos selecionados realizados pelos beneficiários cadastrados nos programas, foi evidenciado o aumento do número total, da média per capita e do custo médio gasto por beneficiário, em Curitiba e Goiânia, entre 2004 e 2006, com redução desses valores em 2007 (Tabela 2).

Os resultados obtidos em relação ao número de exames e procedimentos selecionados per capita no período analisado, assim como o custo médio por beneficiário, tanto em Curitiba quanto em Goiânia, foram estatisticamente significativos (p-valor $<0,001)$ (Tabela 2).

Tabela 1. Número total de consultas, média per capita e custo médio entre os beneficiários cadastrados nos programas para prevenção de doenças cardiovasculares. Curitiba e Goiânia, 2004 a 2007

\begin{tabular}{|c|c|c|c|c|c|c|}
\hline \multirow[b]{2}{*}{ Capital } & \multirow[b]{2}{*}{ Ano } & \multicolumn{3}{|c|}{ Consultas realizadas $^{*}$} & \multicolumn{2}{|c|}{ Custo médio por beneficiário ${ }^{* *}$} \\
\hline & & $\mathrm{N}^{\mathrm{o}}$ total & $\begin{array}{c}\text { Média por } \\
\text { beneficiário }\end{array}$ & $\begin{array}{l}\text { Teste de } \\
\text { Wilcoxon }\end{array}$ & Valor $(\mathrm{R} \$)$ & $\begin{array}{l}\text { Teste de } \\
\text { Wilcoxon }\end{array}$ \\
\hline \multirow{4}{*}{ Curitiba } & 2004 & 1960 & 3,42 & \multirow{4}{*}{$<0,001$} & 126,96 & \multirow{4}{*}{$<0,001$} \\
\hline & 2005 & 1807 & 3,33 & & 130,92 & \\
\hline & 2006 & 1842 & 3,31 & & 137,47 & \\
\hline & 2007 & 1744 & 2,69 & & 107,96 & \\
\hline \multirow{4}{*}{ Goiânia } & 2004 & 852 & 3,23 & \multirow{4}{*}{$<0,001$} & 125,08 & \multirow{4}{*}{$<0,001$} \\
\hline & 2005 & 840 & 3,54 & & 141,76 & \\
\hline & 2006 & 729 & 3,01 & & 121,30 & \\
\hline & 2007 & 464 & 1,77 & & 66,75 & \\
\hline
\end{tabular}

*Clínico geral, cardiologista, endocrinologista e nutricionista. ${ }^{*}$ Valores de 2004, 2005 e 2006 deflacionados com base no IGP-DI de dezembro de 2007

Tabela 2. Número total de exames e procedimentos selecionados, média per capita e custo médio entre os beneficiários cadastrados nos programas para prevenção de doenças cardiovasculares. Curitiba e Goiânia, 2004 a 2007

\begin{tabular}{|c|c|c|c|c|c|c|}
\hline \multirow{2}{*}{ Capital } & \multirow{2}{*}{ Ano } & \multicolumn{3}{|c|}{$\begin{array}{l}\text { Exames e procedimentos } \\
\text { selecionados }\end{array}$} & \multicolumn{2}{|c|}{ Custo médio por beneficiário ${ }^{* *}$} \\
\hline & & $N^{0}$ total & $\begin{array}{l}\text { Média por } \\
\text { beneficiário }\end{array}$ & $\begin{array}{l}\text { Teste de } \\
\text { Wilcoxon }\end{array}$ & Valor $(\mathbf{R} \$)$ & $\begin{array}{l}\text { Teste de } \\
\text { Wilcoxon }\end{array}$ \\
\hline \multirow{4}{*}{ Curitiba } & 2004 & 2339 & 16,77 & \multirow{4}{*}{$<0,001$} & 585,43 & \multirow{4}{*}{$<0,001$} \\
\hline & 2005 & 2402 & 22,00 & & 698,11 & \\
\hline & 2006 & 2405 & 22,84 & & 759,12 & \\
\hline & 2007 & 2211 & 18,89 & & 616,51 & \\
\hline \multirow{4}{*}{ Goiânia } & 2004 & 1054 & 19,06 & \multirow{4}{*}{$<0,001$} & 731,00 & \multirow{4}{*}{$<0,001$} \\
\hline & 2005 & 1064 & 20,91 & & 882,69 & \\
\hline & 2006 & 1070 & 23,43 & & 959,91 & \\
\hline & 2007 & 986 & 18,02 & & 744,03 & \\
\hline
\end{tabular}

*Ácido úrico, clearance de creatinina, colesterol total, HDL-c, LDL-c, creatinina, ECG, glicose, hemoglobina glicosilada, hemograma, lipidograma completo, microalbuminúria, potássio, EAS, triglicerídeos, holter-24 horas, MAPA-24 horas, tilt teste, ergoespirometria ou teste cardiopulmonar de exercício completo e teste ergométrico. ${ }^{* *}$ Valores de 2004, 2005 e 2006 deflacionados com base no IGP-DI de dezembro de 2007 


\section{Análise da utilização de internações por causas} selecionadas

A Tabela 3 demonstra que o número de beneficiários cadastrados em Curitiba que tiveram internações por causas selecionadas diminuiu no período analisado. No entanto, a média de internações por beneficiário foi de 1,56 em 2004, diminuiu em $2005(1,00)$ e voltou a aumentar em $2006(1,25)$, chegando a 2,22 em 2007, resultados estes estatisticamente significativos (p-valor $<0,05$ ). O custo médio por beneficiário também foi maior em 2007 em relação aos demais anos analisados ( $\mathrm{R}$ 9.905,56), porém esses resultados não apresentaram significância estatística (p-valor $>0,05)$ (Tabela 3).

Quanto ao número médio de internações por beneficiário cadastrado em Goiânia, ocorreu aumento em 2005 quando comparado a 2004 (2,00 e 1,40, respectivamente), com redução em 2006 e 2007 ( 1,00 e 1,17, respectivamente). O custo médio por beneficiário aumentou de 2004 a 2006 e diminuiu em 2007. Tanto os resultados referentes à média de internações por beneficiário quanto o custo médio não apresentaram significância estatística ( $\mathrm{p}$-valor $>0,05)$ (Tabela 3 ).

\section{DISCUSSÃO}

Segundo Ribeiro et al. ${ }^{32}$, a elevada prevalência das DC é responsável por altos custos diretos e indiretos, impondo desafios para o setor de saúde e para as políticas públicas para redução dos fatores de risco. Dessa forma, estratégias de saúde pública têm sido implantadas como forma de enfrentamento dessas doenças a partir do enfoque de risco, traduzindo a tentativa de mudança do modelo de assistência prestada à população. Nesse enfoque, a ESF possui papel fundamental na consolidação das ações de promoção da saúde e de prevenção de doenças.

A hipótese da presente pesquisa era que, diante do desenvolvimento de um programa de prevenção de DC, as quantidades de consultas e exames realizados pelos indivíduos participantes aumentariam, e que o número de internações diminuiria a longo prazo. Neste estudo, verificou-se a redução do número médio de consultas por beneficiário cadastrado, enquanto o número de exames per capita aumentou. Em Curitiba, ocorreu aumento do número médio de internações por beneficiário, e, em Goiânia, redução.

A redução do número total de consultas e da média por beneficiário cadastrado, no período de 2004 a 2007, assim como a redução do custo médio per capita, apresentou forte associação estatística.

O aumento do número médio de consultas por beneficiário, observado em Goiânia no ano de 2005, provavelmente, deve-se à implantação da ESF nessa capital no ano de 2003, o que levou à inscrição de novos beneficiários e acarretou um aumento do número de consultas realizadas. Nos anos subsequentes, a gestão da assistência prestada a esse grupo pode ter proporcionado a redução da quantidade de consultas per capita, pelo fato de os programas permitirem um acompanhamento mais próximo e programado da assistência; assim, à medida que esses indivíduos obtêm um perfil de saúde e dos fatores de risco para DC dentro dos parâmetros de normalidade, diminui-se o número necessário de consultas durante $o$ ano.

A redução do número de consultas total e per capita indica que a participação em programas de prevenção de DC viabiliza o acompanhamento mais próximo dos indivíduos e a programação do número de consultas de acordo com o perfil de saúde. A presença de uma equipe própria para a coordenação da atenção à saúde faz com que o beneficiário tenha mais confiança nos profissionais, com o fortalecimento do vínculo, $\mathrm{e}$ não precise realizar várias consultas com diferentes profissionais da mesma especialidade.

Cabe ainda frisar que o desenvolvimento de trabalhos de educação em saúde, voltados para o autocuidado, também

Tabela 3. Número de beneficiários cadastrados que foram internados por causas selecionadas, número médio de internações e custos por beneficiário entre os indivíduos cadastrados nos programas para prevenção de doenças cardiovasculares. Curitiba e Goiânia, 2004 a 2007

\begin{tabular}{|c|c|c|c|c|c|c|}
\hline \multirow[b]{2}{*}{ Capital } & \multirow[b]{2}{*}{ Ano } & \multicolumn{3}{|c|}{ Internações ${ }^{*}$} & \multicolumn{2}{|c|}{ Custo médio por beneficiário ${ }^{\star *}$} \\
\hline & & $\mathrm{N}^{\circ}$ total & $\begin{array}{c}\text { Média por } \\
\text { beneficiário }\end{array}$ & $\begin{array}{c}\text { Teste de } \\
\text { Wilcoxon }\end{array}$ & Valor (R\$) & $\begin{array}{l}\text { Teste de } \\
\text { Wilcoxon }\end{array}$ \\
\hline \multirow{4}{*}{ Curitiba } & 2004 & 16 & 1,56 & \multirow{4}{*}{0,017} & 3905,10 & \multirow{4}{*}{0,322} \\
\hline & 2005 & 10 & 1,00 & & 6630,58 & \\
\hline & 2006 & 8 & 1,25 & & 6683,40 & \\
\hline & 2007 & 9 & 2,22 & & 9905,56 & \\
\hline \multirow{4}{*}{ Goiânia } & 2004 & 5 & 1,40 & \multirow{4}{*}{0,457} & 3096,64 & \multirow{4}{*}{0,611} \\
\hline & 2005 & 8 & 2,00 & & 4537,36 & \\
\hline & 2006 & 5 & 1,00 & & 5568,71 & \\
\hline & 2007 & 6 & 1,17 & & 2239,82 & \\
\hline
\end{tabular}

*AVC, crise hipertensiva, diabetes mellitus, hipertensão maligna, infarto agudo do miocárdio, insuficiência cardíaca, insuficiência coronariana aguda, insuficiência renal crônica, revascularização do miocárdio, tratamento da obesidade e cirurgia de obesidade mórbida. ${ }^{* *}$ Valores de 2004,2005 e 2006 deflacionados com base no IGP-DI de dezembro de 2007 
contribuem para a diminuição da necessidade de consultas e de procura pelos profissionais de saúde.

Pesquisa qualitativa realizada por Reis e Püschel ${ }^{33}$ evidencia as convergências e contradições na implementação da ESF em uma operadora de plano de saúde da modalidade de autogestão, no município de São Paulo, a partir da percepção dos profissionais. Foi evidenciado que o conceito de saúde da família parece transitar entre o modelo hegemônico, curativo, e o modelo idealizado de atenção integral à saúde com bases nas ações de promoção da saúde, porém vinculadas sempre à prevenção de doenças, ao trabalho multidisciplinar e à lógica da diminuição dos custos do sistema.

Jorge $^{34}$ considera positiva a implantação de programas de promoção e de prevenção mesmo que as operadoras de planos de saúde sejam motivadas pelo componente da eficiência e redução de custos, pois a adoção dessa nova proposta e de seus pressupostos irá pressionar pela construção de novas estratégias assistenciais que possam orientar a construção de um novo modelo de atenção à saúde.

O acompanhamento mais próximo dos beneficiários por meio dos programas pressupõe o aumento do número de exames realizados, o que foi demonstrado nas análises, entre 2004 e 2006, com a consequente elevação dos respectivos custos.

A redução do número total de exames e de procedimentos selecionados, da média per capita e do custo médio por beneficiário, em ambas as capitais, no ano de 2007, em comparação aos anos anteriores, que apresentaram tendência de aumento desses valores, pode ter ocorrido devido aos seguintes aspectos: introdução de algum mecanismo de gestão da clínica, evitando a realização de exames e procedimentos desnecessários; e possibilidade de os exames e procedimentos selecionados terem alcançado cobertura e resultados que permitiram sua realização em períodos mais espaçados.

Confrontando os comportamentos observados em ambas as capitais, verificou-se que, em Curitiba, o número de beneficiários internados por causas selecionadas diminuiu no período analisado. Em contrapartida, o número médio de internações por beneficiário aumentou, o que foi estatisticamente significativo ( $\mathrm{p}$-valor $<0,05$ ). $\mathrm{O}$ aumento do valor médio gasto com cada indivíduo, entre 2004 e 2007, não foi significativo do ponto de vista estatístico.

É relevante destacar que, diante da impossibilidade de utilizar a CID para a seleção das causas de internação, alguns dados podem estar subestimados, tendo em vista que muitas internações para realização de procedimentos cirúrgicos podem ter sido motivadas por DC ou pelas demais doenças selecionadas, não identificadas na base de dados devido ao precário preenchimento da CID.
Outro aspecto importante é que o tempo de participação no programa pode não ter sido suficiente para causar impactos na redução do número de internações, pois muitos desses beneficiários foram inscritos justamente por terem problemas cardiovasculares, serem idosos e/ou participarem do programa de assistência domiciliar, o que por si só aumenta a chance de internação. Tais resultados apontam para a importância de realizar novos estudos que tenham maior tempo de acompanhamento dos beneficiários participantes desses programas.

Revisão sistemática realizada por Shroufi et al. ${ }^{35}$ confirma a evidência de custo-efetividade de intervenções voltadas para a prevenção de DC, com ressalva para a necessidade de mais estudos em países de média e baixa renda.

O monitoramento e a avaliação dos resultados constituem desafios a serem enfrentados pelas operadoras que realizam programas de promoção e de prevenção. A lógica do mercado de saúde, voltada para o pagamento de procedimentos, traz consigo a ausência de informações relativas ao perfil epidemiológico da carteira de beneficiários, dificultando o planejamento de um programa voltado para as necessidades de saúde da população.

$\mathrm{Na}$ opinião de Silveira ${ }^{36}$, é fundamental investir e aprimorar os sistemas de informação, para que sejam capazes de fornecer informações sobre as condições de saúde dos beneficiários das operadoras de planos de saúde, viabilizem a obtenção permanente de dados e a avaliação de séries históricas, e, principalmente, subsidiem a tomada de decisão.

Segundo Silva ${ }^{37}$, os desafios da modelagem tecnoassistencial no setor suplementar de saúde são a oferta de redes de cuidados articuladas, focadas nas necessidades priorizadas epidemiologicamente, e a abertura para as subjetividades dos beneficiários. Além disso, o autor acredita que o uso de tecnologias leves, a melhora nas escolhas das tecnologias duras e leve-duras, a criação de vínculo e uma competente coordenação dos cuidados em rede são apostas para a obtenção de melhores resultados.

Sabe-se que as empresas de planos de saúde também buscam, por meio do desenvolvimento de programas de promoção da saúde e prevenção de doenças, a redução dos custos assistenciais, no entanto esse objetivo precisa estar alinhado com as políticas de saúde e com a necessidade de aumentar a qualidade da atenção à saúde.

\section{CONCLUSÃO}

Sem a pretensão de generalizar os resultados demonstrados, o presente estudo de caso objetiva contribuir para a diminuição de uma lacuna existente no que tange à ausência de estudos que avaliem os resultados obtidos por meio do desenvolvimento de 
programas de promoção da saúde e de prevenção de doenças, principalmente no setor privado de assistência à saúde.

À guisa de conclusão, a responsabilização pelo beneficiário, a prestação de serviços orientados pelas necessidades de saúde, a adoção de equipes multidisciplinares, o monitoramento permanente dos resultados e a qualidade da assistência prestada devem ser os princípios norteadores da conduta do mercado de saúde suplementar, com vistas à obtenção de impactos positivos na saúde dos beneficiários e à construção de um novo modelo de atenção no setor.

Nesse sentido, torna-se relevante avançar no debate em torno da promoção da saúde e da prevenção de doenças na saúde suplementar, com a realização de mais estudos quantitativos e qualitativos, além da necessidade de conciliar a prática do setor privado com as políticas empreendidas pelo MS, a fim de contribuir de forma efetiva para a melhoria das condições de saúde da população brasileira.

\section{REFERÊNCIAS}

1. Agência Nacional de Saúde Suplementar. Resolução Normativa ${ }^{\circ} 94$ de 23 de março de 2005. Dispõe sobre os critérios para o diferimento da cobertura com ativos garantidores da provisão de risco condicionada à adoção, pelas operadoras de planos de assistência à saúde, de programas de promoção à saúde e prevenção de doenças de seus beneficiários. Diário Oficial da União, Brasília, 24 de março de 2005.

2. Agência Nacional de Saúde Suplementar. Promoção da saúde e prevenção de riscos e doenças no setor de assistência privada à saúde: atuação da Agência Nacional de Saúde Suplementar. In: Agência Nacional de Saúde Suplementar. Atenção à saúde no setor suplementar: evolução e avanços do processo regulatório. Rio de Janeiro; 2009. p. 29-41.

3. Agência Nacional de Saúde Suplementar. Instrução Normativa Conjunta nº 001 de 30 de dezembro de 2008 da Diretoria de Normas e Habilitação das Operadoras - DIOPE e da Diretoria de Normas e Habilitação dos Produtos - DIPRO. Dispõe sobre o cadastramento, monitoramento e os investimentos em programas de promoção da saúde e prevenção de riscos e doenças por parte das operadoras de planos privados de assistência à saúde. Diário Oficial da União, Brasília, 31 de dezembro de 2008.

4. Agência Nacional de Saúde Suplementar. Resolução Normativa n ${ }^{\circ}$ 264, de 19 de agosto de 2011. Dispõe sobre Promoção da Saúde e Prevenção de Riscos e Doenças e seus Programas na saúde suplementar. Diário Oficial da União, Brasília, 22 de agosto de 2011.

5. Agência Nacional de Saúde Suplementar. Resolução Normativa $n^{\circ} 265$, de 19 de agosto de 2011. Dispõe sobre a concessão de bonificação aos beneficiários de planos privados de assistência à saúde pela participação em programas para Promoção do Envelhecimento Ativo ao Longo do Curso da Vida e de premiação pela participação em programas para População-Alvo Específica e programas para Gerenciamento de Crônicos. Diário Oficial da União, Brasília, 22 de agosto 2011.

6. Agência Nacional de Saúde Suplementar. Instrução Normativa no 35 , de 19 de agosto de 2011 da Diretoria de Normas e Habilitação dos Produtos DIPRO. Regulamenta a $\mathrm{RN} \mathrm{n}^{\circ} 264$, de 19 de agosto de 2011, dispondo sobre o acompanhamento dos programas para Promoção da Saúde e Prevenção de Riscos e Doenças desenvolvidos pelas operadoras de planos privados de assistência à saúde. Diário Oficial da União, Brasília, 22 de agosto de 2011.

7. Agência Nacional de Saúde Suplementar. Instrução Normativa no 36, de 19 de agosto de 2011 da Diretoria de Normas e Habilitação dos Produtos DIPRO. Dispõe sobre os procedimentos para cumprimento da Resolução Normativa $^{\circ} 265$, de 19 de agosto de 2011. Diário Oficial da União, Brasília, 22 agosto de 2011 .

8. Agência Nacional de Saúde Suplementar. Instrução Normativa Conjunta $n^{\circ} 7$ de 23 de novembro de 2012. Dispõe sobre o cadastramento, o monitoramento e os investimentos em programas para promoção da saúde e prevenção de riscos e doenças por parte das operadoras de planos privados de assistência à saúde. Diário Oficial da União, Brasília, 28 de novembro 2012.
9. Agência Nacional de Saúde Suplementar. Manual técnico para promoção da saúde e prevenção de riscos e doenças na saúde suplementar. 4. ed. Rio de Janeiro; 2011.244 p.

10. Agência Nacional de Saúde Suplementar. Cartilha para a modelagem de programas para promoção da saúde e prevenção de riscos e doenças. Rio de Janeiro; 2011. 80 p.

11. Agência Nacional de Saúde Suplementar. Plano de cuidado para idosos na saúde suplementar. Rio de Janeiro; 2012. 62 p.

12. Organização Mundial da Saúde. World Health Professions Alliance. Agência Nacional de Saúde Suplementar. Associação Médica Brasileira. Plano de melhoria da saúde: um guia para pacientes e público em geral. Rio de Janeiro: Agência Nacional de Saúde Suplementar; 2012. 17 p.

13. Organização Mundial da Saúde. World Health Professions Alliance. Agência Nacional de Saúde Suplementar. Associação Médica Brasileira. Cartão plano de melhoria da saúde. Rio de Janeiro: Agência Nacional de Saúde Suplementar; 2012.

14. Agência Nacional de Saúde Suplementar. Planos odontológicos: evolução, desafios e perspectivas para a regulação da saúde suplementar. Rio de Janeiro: Agência Nacional de Saúde Suplementar; 2009. 152 p.

15. Agência Nacional de Saúde Suplementar. Avanços da oncologia na saúde suplementar. Rio de Janeiro; 2014. 40 p.

16. Organização Pan-Americana da Saúde. Agência Nacional de Saúde Suplementar. Promoção da saúde e prevenção de riscos e doenças na saúde suplementar brasileira: resultados do laboratório de inovação. Brasília: OPAS; 2014. $112 \mathrm{p}$

17. Agência Nacional de Saúde Suplementar. Agenda Regulatória: um compromisso da ANS com a sociedade [Internet]. [citado em 2013 jun 18]. Disponível em: http://www.ans.gov.br/aans/transparencia-institucional/2024-agendaregulatoria-temas-envolvidos2\#eixo1

18. Médici AC. Aspectos teóricos e conceituais do financiamento das políticas de saúde. In: Piola SF, Vianna SM, organizadores. Economia da saúde: conceito e contribuição para a gestão da saúde. Brasília: Instituto de Pesquisa Econômica Aplicada; 1995. p. 23-70.

19. Silva Jr AG. Modelos tecnoassistenciais em saúde: o debate no campo da saúde coletiva. 2. ed. São Paulo: Hucitec; 2006. 143 p.

20. Brasil. Lei no. 9.656, de 3 de junho de 1998. Dispõe sobre os planos privados de assistência à saúde. Diário Oficial da União, Brasília, 4 de junho de 1998.

21. Agência Nacional de Saúde Suplementar. Caderno de Informação da Saúde Suplementar [recurso eletrônico]: beneficiários, operadoras e planos. Rio de Janeiro; 2015. 63 p. 
22. Almeida C. O mercado privado de serviços de saúde no Brasil: panorama atual e tendências da assistência médica suplementar. Brasília: Instituto de Pesquisa Econômica Aplicada; 1998. 49 p.

23. Derengowski MG. Nota introdutória sobre estrutura de mercado, concentração e mercados relevantes. In: BRASIL. Ministério da Saúde. Agência Nacional de Saúde Suplementar. Regulação e saúde: documentos de apoio ao fórum de saúde suplementar de 2003. Rio de Janeiro: Ministério da Saúde; 2004. p. 65-92. v. 3, t. 1. (Série B. Textos básicos de saúde. Regulação e saúde).

24. União Nacional das Instituições de Autogestão em Saúde. Autogestão em saúde no Brasil 1980-2005: história da organização e consolidação do setor. São Paulo; 2005. 94 p.

25. Coombes Y, Thorogood M. Introduction. In: Thorogood M, Coombes Y. Evaluating health promotion: practice and methods. New York: Oxford University Press; 2000. p. 3-10.

26. Hartz ZMA, organizador. Avaliação em saúde: dos modelos conceituais à prática na análise da implantação de programas. Rio de Janeiro: Fiocruz; 1997. $131 \mathrm{p}$.

27. Brasil. Ministério da Saúde. Secretaria de Atenção à Saúde. Departamento de Atenção Básica. Prevenção clínica de doença cardiovascular, cerebrovascular e renal crônica do Ministério da Saúde. Brasília: Ministério da Saúde; 2006. 56 p. (Cadernos de Atenção Básica, 14).

28. World Health Organization. Prevention of cardiovascular disease: guidelines for assessment and management of cardiovascular risk. Geneva; 2007. 86 p.

29. Fundação Getúlio Vargas. Conjuntura estatística. Conjunt Econ. 2008; 62(6), II-XIX.

30. Brasil. Ministério da Saúde. Secretaria de Atenção à Saúde. Departamento de Regulação, Avaliação e Controle de Sistemas. Diretrizes para a programação pactuada e integrada da assistência à saúde. Brasília: Ministério da Saúde; 2006. $148 \mathrm{p}$.
31. Associação Médica Brasileira. Classificação Brasileira Hierarquizada de Procedimentos Médicos. 4. ed. São Paulo; 2005. 197 p.

32. Ribeiro AG, Cotta RMM, Ribeiro SMR. A promoção da saúde e a prevenção integrada dos fatores de risco para doenças cardiovasculares. Cien Saude Colet. 2012;17(1):7-17. http://dx.doi.org/10.1590/S1413-81232012000100002. PMid:22218533.

33. Reis ML, Püschel VAA. Estratégia de Saúde da Família no Sistema de Saúde Suplementar: convergências e contradições. Rev Esc Enferm USP. 2009; 43(Esp 2):1308-13.

34. Jorge AO. Refletindo sobre a caminhada. In: Agência Nacional de Saúde Suplementar. Panorama das ações de promoção da saúde e prevenção de riscos e doenças no setor suplementar de saúde. Rio de Janeiro: Agência Nacional de Saúde Suplementar; 2008. p. 77-87.

35. Shroufi A, Chowdhury R, Anchala R, Stevens S, Blanco P, Han T, et al. Cost effective interventions for the prevention of cardiovascular disease in low and middle income countries: a systematic review. BMC Public Health. 2013;13:285. http://dx.doi.org/10.1186/1471-2458-13-285. PMid:23537334.

36. Silveira LS. Prevenção de doenças e promoção da saúde: diferenciais estratégicos na conjuntura da saúde suplementar [dissertação]. Rio de Janeiro (RJ): Escola Nacional de Saúde Pública Sergio Arouca, Fundação Oswaldo Cruz; 2004.

37. Silva Jr AG. Promoção à saúde e mudanças nas práticas da saúde suplementar. In: Agência Nacional de Saúde Suplementar. Panorama das ações de promoção da saúde e prevenção de riscos e doenças no setor suplementar de saúde. Rio de Janeiro: Agência Nacional de Saúde Suplementar; 2008. p. 71-76.
Recebido em: Jan. 15, 2016

Aprovado em: Jun. 14, 2016 